\title{
Interpretivistic Conception of Quantification: Tool for Enhancing Quality of Life?
}

\author{
Denis Larrivee ${ }^{1 *}$, Adriana Gini ${ }^{2}$ \\ ${ }^{1}$ Educational Outreach Office, Roman Catholic Diocese of Charleston, Charleston, USA \\ ${ }^{2}$ Neuroradiology, Neuroscience Department, San Camillo Forlanini Medical Center, Rome, Italy \\ Email: *sallar1@aol.com
}

Received May $6^{\text {th }}, 2013$; revised June $6^{\text {th }}, 2013$; accepted June $13^{\text {th }}, 2013$

Copyright (C) 2013 Denis Larrivee, Adriana Gini. This is an open access article distributed under the Creative Commons Attribution License, which permits unrestricted use, distribution, and reproduction in any medium, provided the original work is properly cited.

\begin{abstract}
Quality of life is fast becoming the standard measure of outcome in clinical trials, residential satisfaction, and educational achievement, to name several social settings, with the consequent proliferation of assessment instruments. Yet its interpretation and definition provoke widespread disagreement, thereby rendering the significance of quantification uncertain. Moreover, quality, or qualia, is philosophically distinct from quantity, or quantitas, and so it is unclear how quantification can serve to modulate quality. Is it thus possible for quantification to enhance quality of life? We propose here that an interpretivistic conception of quantification may offer a more valid approach by which to address quality of life in sociological research.
\end{abstract}

Keywords: Interpretivism; Quantification; Dialectical; Quality of Life

\section{Introduction}

Given the significance and widespread cultural interest in quality of life issues, the pursuit of quantification so as to enhance life's quality has also assumed proportional significance (Ware \& Gandek, 1998). Quantification, pursued in this context, is intended to identify and evaluate parameters judged to impact quality of life favorably, and to attain, thereby, predictive status. While it is generally assumed that such parameters will modify quality of life, they do not in themselves constitute the experiential dimension so altered. Quantification is thus rendered indirect and dependent upon quality of life definitions for its validation.

Definitional clarity is by no means easily attainable, and numerous variants have been proposed (Chung, Killingworth, \& Nolan, 1998). Quality of life has been related to performance in employment and social spheres (Kahneman \& Deaton, 2010), affective enjoyment, intellectual fulfillment, or economic status (Rappaport, 2008), to name several different variables. In circumstances of hardship, response to physical or psychological impairments has also been evaluated. The diversity and ambiguity of definitions have generated widespread disagreement rendering interpretations of quantitative assessments uncertain and restrictive.

Recourse to empirical verification in order to validate qualitative social phenomena derives from the thought of SaintSimon and Comte and their development of positivistic philosophy in the mid nineteenth century (Comte, 2011). According to positivism, scientific investigation provides the singularly valid form of inquiry. Its claims rest upon the quantification of facts, which are the sole objects of human knowledge. The aim

\footnotetext{
"Corresponding author.
}

of positivism is thus to construct general principles from empirically gathered data that would undergird an understanding of personal conduct and social organization.

By contrast, the philosophy of interpretivism, often designated as anti-positivism for its reactionary stance to positivism, claimed that social sciences were not amenable to empirical evaluation, unlike the natural sciences (Kim, 2006). In the view of interpretivists, social research was best performed by focusing on the subjective interpretation of social actions of those being studied. A cluster of philosophical disciplines, drawn from Hegelians, Marxians, and neo-Kantians rejected Comtean sociological positivism, introducing in its stead verstehende, or sociological antipositivism, at the turn of the twentieth century (Kim, 2006). It was proposed that sociology could be described as a science in so far as its ability to methodologically identify causal relationships of human social action, an identification that would be unavailable empirically. Thus, antipositivism's intent was to provide causal explanation of the way in which social action proceeds, and, analogously, in which it was subjectively meaningful.

Approaches to sociological research have since divided along positivist and antipositivist lines (Leitch, Hill, \& Harrison, 2010), with most sociological work in the United States adopting empirical validation as the principal schema within which to articulate and undergird social hypotheses. Despite this preference, conclusions based on the positivist paradigm continued to suffer from the objections originally raised by early twentieth century antipositivism and subsequently expressed in the latter twentieth century by intellectual descendants of the Frankfurt School in such forms as Critical Theory and reflective disclosure (Habermas, 2011).

Given the impact of definitional ambiguity on assessment 
tools in current quality of life studies, it seems to us that an interpretivistic approach is needed to reconfigure the use of quantification as an exploratory medium for quality of life research. We consider here the process of quantification as experientially mediating, and thereby one subjectively modulating quality of life. Three aspects of quantification are considered: order, information quantity, and the presence of intrinsic dialectical elements. We contend that while all three are contributory it is the presence of an intrinsic dialectic that is essential to the operational engagement of the agent.

\section{Quality and Quantification: A Categorical Foundation}

Conceptual definitions of quality and quantity enjoy ancient lineage and emerge from Aristotelian designations. Quality and quantity are philosophically distinct and constitute fundamental categories.

Quality, or qualitas, is regarded as an attribute or a property of an object (Aristotle, 2007). It is an expression of the stable unity of an object's structure, which makes it distinct from other objects. As such it may be compared relative to other qualities, such as hard in relation to soft. Quantity, or quantitas, by contrast, may be likened to a mathematical set. Homogeneous objects may be grouped together to constitute a set and, if they can be counted, they are considered finite. The basis of quantitas is thus the objective discreteness of things, which is expressed numerically. Quantitas may also be used to express magnitude, a continuously changing variable. Quantitas may not be used to distinguish between qualities. It is not possible to relate three pomegranates and two figs, for example (Aristotle, 2007).

Quantitas may, however, be used to relate homogeneous objects. Quantification, then, permits the comparison of sets of like objects. By quantification it is possible to express the external formal relation of parts, properties, connections and so on of objects. Thus, evaluation of relational order necessitates quantification. A length "A" may be greater or lesser than length " $B$ ", but the magnitude relationship between lengths " $A$ " and " $\mathrm{B}$ " will only become apparent upon the completion of the process of quantification.

Relations between quantities may be more or less complex. A simple relationship may involve only a direct relationship of the magnitude of two differing quantities. A more complex relationship may involve the relationship between a third quantity and its dependency upon two other quantities. "C", for example, may be a contingent quantity depending upon the valuation of "A" and " $B$ ", and can be expressed as " $C$ " = " $A$ " + " $B$ ". That is, in the absence of " $A$ " or " $B$ ", "C" does not manifest itself. Analogously, relationships of progressively greater dependency can be elaborated. In either case, simple or complex, an evaluation of relationship can only proceed if quantification has been performed.

The subjective role of quantification becomes apparent when we consider its function in assessing order. Ordered arrangements retain greater intrinsic appeal than disordered arrangements, presumably because they enable the prediction and response to that which is comprehensible.

Thus, quantification, in enabling us to assess whether the relations within and between sets constitute ordered arrangements, interjects a subjective component in the evaluative process.
Definitionally, order is characterized by three elements: complexity, the presence of an ordering principle, and the degree of conformity of the elements to an ordering principle; hence, an arrangement exhibiting these features would be considered ordered. Clearly, order requires the presence of complexity. It cannot be present in a single element for it necessitates the existence of relations, which can only reside amongst a multitude of members. Order must also proceed according to a plan, that is, an ordering principle that defines the relations amongst constituent elements. In its simplest form an ordering principle may dictate a single feature governing members of a set, such as books on a shelf. An ordering principle may be more or less complex, however, and govern numerous features of its sets. Finally, order necessitates conformity to the ordering principle, else the set would be regarded as disordered. In the extremes no member of the set would adhere to the ordering principle, in which case the set would be considered completely disordered. Conversely, it may conform entirely, in which case the set would be completely ordered. Thus, order may be discerned through the characterization of an underlying organizing principle to which there is more or less conformity.

When sets exhibit a high degree of conformity to the ordering principle, their order is said to retain a mathematical order (Russell, 1919). Mathematical order is characterized by complete coherence amongst set members, all of which adhere to the designated mathematical relationship. For example, the order of points on a line is essential to a linear characterization. Likewise, the concept of limit, is a progressive concept of serial placement. In each case order lies in the consistency of relationships amongst the members of the sets. A fixed point may precede or follow another and the relationship between both points will be given by the placement and interval separating the two. Analogously, numerous other such relationships also exist. The discernment of a mathematically defined order would thus imply a highly ordered state in a set so evaluated, one for which there is a resident, subjective appeal by virtue of the apprehension of its order.

While it is generally agreed that ordered states are preferable to disordered ones, it may yet remain true that as the sole contributory factor order offers little subjective meaning for an observer. A set composed of a single feature, for example, such as the set of names of individuals inhabiting a single geographical locus, would certainly have little meaning beyond the single, defining feature of the set. A paucity of information would, accordingly, yield a dearth of meaning. Sets with little information content are simply not very meaningful. Extrapolating therefrom, meaning seems to bear a positive relationship to the informational content of a set, and more meaning seems to be extracted from sets that have higher information content.

Is this sort of conclusion always valid? The question may be answered in part by recourse to information theory (Shannon, 1948), which can help in determining an upper bound for a relational dependence of subjective meaning on increased information content. As described by the theory, information content displays dependencies on entropy, a thermodynamic measure of disorder, and is inversely related to redundancy. The theory predicts that more information is yielded by systems in which there is greater dissimilarity between members of a set. Taken to its limit, a system in which there is no coherence ought to yield a maximum degree of information. Yet, this is also the definition of a completely disordered system, and there is undoubtedly general concurrence that complete disorder contains 
little that would be meaningful to an observer. Thus, while it would seem that greater informational content characterizes a system generating greater meaning, there are also limits to the extent to which this relationship may be extrapolated. Apparently, some degree of intrinsic order is also needed. The situation is reminiscent of various theoretical efforts which have explored the nature of aesthetic preferences (Birkhoff, 1933). These theories generally proffer the thesis that aesthetically pleasing objects, such as art, retain a measure of order, while simultaneously displaying a high degree of variability. The presence of coherent order, when conjoined to significant complexity in an object, is said to generate an appeal that is immensely meaningful to an observer.

Nevertheless, increasing the informational content of an ordered set is alone unlikely to suffice for provoking subjectively meaningful responses. Consider, for example, the case of tossing one or several die. A single dice may generate six different outcomes, whereas several are capable of generating six raised to the power of the number of die tossed. Clearly, the second situation is productive of a quantitatively greater information content. Yet, it is not necessarily more meaningful. Meaning, then, is not dictated solely by the quantity of information content.

Some distinction is therefore required with respect to the type of information that is yielded by the set, a qualitative distinction that is constitutive of the set, yet newly operative in its subjective engagement. A qualitative analysis is therefore also needed, one that is capable of revealing qualitative distinctions between respective data sets. Such an evaluation can be expected to identify and designate features of the data sets that would enrich their meaning. However, given that the initial quantitative evaluation depends a priori upon qualitative distinctions established by the original ordering principle the qualitative analysis cannot simply restate the original features on which the ordering principle was first structured. A new set of attributes must thus be determined else there would be no new qualitative features.

How might these new attributes be inferred? From where do they originate?

The revelation of new attributes can originate only from new sources of information that are localized within the set, since it is the set that is the source of meaning. Externally applied attributes would, by contrast, represent a source of meaning imposed upon the set. Since any new attributes would need to be disclosed by means of an evaluation of the original set, we may ask whether the initial quantitative evaluation is adequate to reveal the presence of novel qualitative features? A cursory examination of our prior examples would suggest not. Once we have quantified our geographically restricted set of names and established their alphabetical order, for example, there is little new information that would suggest novel population characteristics. The same may be inferred from our example of die casting. Thus, certain types of information sets seem incapable of yielding further qualitative distinctions beyond those initially established by the ordering principle.

What characterizes these qualitatively "poor" sets? Implicitly, an inability to yield qualitatively new information characterizes set relationships with little or no interaction. By definition such relations may be described as mutually insensitive. Put another way, the quantitative evaluation does not provoke an additional interpretation of the set by virtue of their indifferent relations.

Do qualitatively "poor” sets exhaust the full range of sets that may be evaluated quantitatively? Are there sets that are qualitatively "rich"? What would characterize them? We expect that these sets, unlike those which are qualitatively "poor", will offer new characterizations of the set information, beyond those dictated by the ordering principle. To do so will require the presence of mutually sensitive relations. Due to their mutual sensitivity, relations between sets can be expected to evoke new qualities in the complementary partners (Lorand, 2000). Consider a work of visual art, for example, in which an artist seeks to highlight a single subject. By juxtaposing the illuminated subject with a dark field the subject acquires a greater intensity of focus, a quality that would not be evident in the absence of the field. The initial quantitative evaluation assesses both the magnitude of the dark and of the light regions. Both complement one another and each is thus sensitive to the other's "influence". Their mutual sensitivity evokes a new quality that would not be evident in the absence of the complementary interaction. This suggests that quantitatively "rich" sets do exist, and that they are characterized by mutually sensitive relations.

The existence of such sets is a more specific representation of the dichotomous interactions broadly distributed in the natural world, yet also observed at social, philosophical, and artistic levels (Adorno, 1970), interactions which have been codified by Hegel (Bristow, 2007). Hegel's dialectical dynamic configures the nature of reality within a web of mutual interactions whose forces provoke a continual alternation in motion between dialectical partners. In our example, taken from art, the dark background emphasizes its contrasting light filled area. The greater the darkness, moreover, the more emphasis the light filled area receives. Its quality of intense focus is amplified and dependent upon the greater quantity of darkness. Thus, the dichotomy of two contrasting areas produces mutually sensitive relations whose intensity is related to the magnitude of the interacting partners. These are made evident through the quantitative evaluation.

The interactive dynamic constitutes a new quality that is the product of the mutual sensitivity of the complementary sets. Since the generation of the new quality as well as the intensity of its relationship is dependent upon the quantitative evaluation of the dialectical partners, the new qualities may be considered to be engendered by the act of quantification.

The transformation that is effected is representational of a categorical shift that displaces the quantitatively structured set elements with the qualitative dynamic of the dialectic. An analogous transformation has been frequently invoked in reference to social and political dynamics, but the law, in fact, springs formally from an analytical conception of the material world (Spirkin, 1983). Dialectical materialists view a materially limited, natural world from the lens of its dynamic character, wherein its constituents are in opposition and thereby rendered perpetually motile. According to the law, the process of development, or transformation, is one in which small and insignificant changes in quantity gradually proceed until an abrupt change to a new form is experienced. Accretion of natural processes thus culminate in the attainment of critical levels, before being succeeded by the emergence of wholly new forms. Transformation as such is temporally conditioned in that the transformation succeeds the quantitative changes but need not be causally initiated by them. Transformation arising from the disclosure of the quantitative evaluation is also conditioned by temporal sequence but, by contrast, appears to be incurred by 
the quantitative process. Contrast, for example, is made manifest by the quantification of numerical differences between data sets which have been placed in opposition. Quantification in this sense bears elements common to dialectical materialism in its transitional and to anti-positivism in its subjectively causal features.

\section{Quantification and Art: An Experiential Paradigm}

Which information sources co-identify with qualitatively "rich" sources? Certainly, relational qualities that might be revealed by quantification are legion. To limit our study we chose to explore the role of quantification in art, since artistic beauty is known to exert a strong and subjective impact on the observer. Specifically, we explored whether the preceding features of order, information content, and resident dialectic may be exhibited in examples taken from visual art, music, or architecture, since it is these features that are likely to prove significant for communicating subjective meaning.

\section{Order}

Art is ordered. All arts adhere to clearly defined principles for the construction of their various compositions. Visual arts, for example, include contrast, rhythm, pattern, balance, emphasis, proportion, variety and harmony (Tersiisky, 2004), which constitute its properties or qualities. The evaluation of these qualities necessitates their quantification. The evaluation of rhythm, for example, permits the determination of repetitive sequences, such as the number and frequency of spatial occurrences. Clearly, many other qualities also require quantification to properly assess their contribution to the compositional structure. Other art forms, such as music and architecture, employ similar qualities in their compositions, with a corresponding need for quantitative evaluation.

The order present in art, moreover, frequently displays mathematical coherence (Schillinger, 1948). Indeed, numerous mathematical descriptions have been articulated for relationships between compositional components. Architectural arts, for example, depend upon geometrical configurations (Salingaros, 2013), and rigorous study of mathematical form is strongly recommended for students of the profession. Geometrical configurations are often related by means of scaling, or by rotational or translational displacements, and when repeated on a hierarchy of scales, generate fractal patterns, which can be described by fractal mathematics. In still another example, musical notes are temporally distributed as components within equally spaced time intervals, or beats, that can be metered. Implicit in all these mathematical descriptions is the existence of highly ordered relationships that underlie the compositional structure of the art form.

Furthermore, while many compositions reflect an order imposed by the artist, in still others order arises as an inherent constituent of the art form itself, one which displays clear origins to mathematically ordered relationships found in nature. Western music, for example, exhibits a particularly close affinity for tonal properties associated with the physics of sound. Musical scales repeat the "same" sounds at higher pitches with identical sounds evoked at higher pitches related by integer multiples of tone frequency. Intermediate tones that form pleasing combinations are typically related by common ratios of frequencies. Fifths, for example, fall at intervals midway between frequencies associated with the initial scale tone, and major thirds at quarter intervals.

\section{High Information Content}

Art conveys high information content (Moles, 1966). At its most fundamental level art is structured to communicate a message. Hence art must retain an information content that is constitutive of the message that is to be delivered. What is the message, and what is the medium of delivery?

That which most nearly distinguishes the artistic composition is its sense of encounter and intimacy (Gadamer, 1986). The work of art intersects with our intelligibility and situates us in a world where our engagement unfurls a continually expanding, relational context (Heidegger, 2008). The breadth of engagement that encompasses such a panorama thereby necessitates a correspondingly adequate informational resource, one that is likely to share multiple origins.

\section{Disclosure of the Intrinsic Dialectic}

Art is self interpretive (Lorand, 2000). Each form is conceptually whole, its elements juxtaposed to generate a single image. The elements maintain fixed relationships, and it is these that define the basic form. Their integrity is requisite. Dispersal, and the consequent compromise to formal integrity, collapses the form, in effect generating multiple new images. The potential for dissolution is thus confirmation of the need for elemental interdependence. It is on this basis that explication of the art form by quantification may proceed.

The form's dependence on juxtapositional stability establishes the proximate relationships of its constituents, be they physical or temporal, and conditions their mutual interpenetration. Their mutual sensitivity, however, is dictated by their oppositional characterization. Proximity thus modulates the intensity of the reciprocative exchange. Their mutual interpretive activity follows from the manifestation of complementarity, initiating their capacity to mutually evoke new features. Quantification in its revelatory role discloses the dialectic that becomes operative through the discernment of the complementary elements.

The interpretative function arises in the dialectic operation as each feature endows its complement with a greater sense of meaning, necessitating the simultaneous presence of the other. In this sense each feature serves to interpret all other features with which it is related. Indeed it is the recognition that constituent elements themselves endow interpretive value that the basis of the rationale behind formal analysis is grounded, and in which the compositional elements are deciphered in conjunction with one another.

A simple illustration from the Bach two part invention number eight provides a case in point. The opening subject is expressed by a motif in which articulated, eighth note spacings alternate between a tonic and sequential intervals of thirds, culminating in the tonic of the next register. A second motif follows in the next bar with a non articulated descent from the upper tonic of sixteenth notes with spacings of whole tone intervals. The second bar simultaneously repeats the opening motif in an octave lower register which serves as counterpoint to the second motif. Several examples of dialectical partners are evident in the pairings of the first and second motives: the ascending and descending cascades, the articulated and nonarticulated expressions, and the contrasting temporal spacing. 
Disclosure of the dialectical partners occurs through the quanitative evaluation of time and pitch, and makes operative the dialectic through the contrast that has been made evident in the disclosure.

Order, high information content, and mutually sensitive relations are thus constitutive of art, and endow art with characteristics that make it a rich source of subjective meaning.

\section{Art as Subjectively Causal}

Is there corroborative support for a causally mediated, subjective influence of art?

In the natural sciences causality may be considered the explication of the relationship between two phenomena and of the dependency of actuation by one phenomenon on the other, a definition that has been largely retained by the social sciences (Moreno \& Martinez, 2006). A definitional corollary is that of temporal asymmetry, understood as the precedence of one phenomena with respect to the other. Proceeding from these definitions, quantification of art may be considered causal, in that it precedes and makes manifest qualities within the art form which would otherwise not be perceived. Numerous studies document the profound extent of the subjective impact of artistic beauty. We will briefly consider what these explorations reveal of the communication of meaning to an observer.

As a subjectively meaningful experience, art significantly impacts cognitive phenomena (Zeki, 1999). Its various effects have been documented with reference to affective, physiological, and developmental dimensions, to name a few (Soslo, 2004; Chatterjee, 2010). How does the human brain process art? How is it reconstructed and analyzed? More pertinently, how are broadly based responses elicited and what salience can be attributed to various features that elicit these responses? To take just one example, the perception of musical sound can evoke a broad spectrum of emotional repertoire, with origins in multiple cognitive centers (Bergeron \& Lopes, 2009).

These studies make implicit the presence of neurobiological underpinnings receptive to the informational content of the art form. When presented with salient stimuli cognitive centers respond in ways that reflect common neural paradigms whose expression is circumscribed, but not impeded, by individual variation. The presentation of various art forms has been correlated with the production of fear in the amygdala, pleasure in the nucleus accumbens, or disgust in the insula (LeDoux, 2002; Zeki, 1999). Socially imbued images may evoke activation of temporo parietal regions. Still other forms, often found in architecture, may activate preferred, evolutionarily constructed developments intended for self preservation (Hildebrand, 1999; Donald, 1991).

Perhaps the most thoroughly investigated circuitry is that associated with visual perception. Artistic imagery, as with all visually processed form, passes to the occipital lobe from the sensory receptive centers of the retina, where informational features are distinguished and extracted prior to their delivery to higher order neural centers. Portrait artistry, for example, appears to employ processing circuitry localized within the fusiform facial area and the right prefrontal cortex (Solso, 2004), a conclusion drawn from the increased activity that is observed in these centers during sketching. Patient elicited, behavioral assessments likewise provide corroborative evidence (Zeki, 1999).
Theorizing from such observations contemporary philosophies of aesthetics have emphasized a nearly exclusive determination of artistic beauty that springs from a subjective mediation (Zeki, 1999). Indeed, the pragmatic facility with which neurological centers can be assessed has served to reinforce these notions, to the detriment of the recognition that any intrinsically objective character may reside within the art form. Correspondingly, this has prompted a focus on research attempting to identify a single neurological center within which comprehensive notions of beauty are formulated (Zeki, 1999).

Extrapolating therefrom art is said to engage inherent structures which are functionally needed to relate to an often capricious external world, a world which may present itself on one occasion as beneficent and on another as destructive. Their development and retention is thought to form the adaptive bulwark within which both flourishing and dissemination propel their arching trajectories (Donald, 1991). Human conscious capacity, it is proposed, shaped the symbolic culture through which conventions, customs, and protorituals elicited the fabrication of large scale social structure, its executive role constituting a central guidance system that permitted the internal management of cognitive phenomena and their selective orientation to a specifically evaluative and strategical function. From this perspective, art may be considered to engage mechanisms which are need based and externally oriented. As such it offers meaning of a principally utilitarian character.

One may ask whether a utilitarian dimension exhausts the full extent of meaning that is conveyed by the art form, a dimension dictated by the evolutionary constructs of a neural landscape. Is arts' subjective meaning limited to a purely aesthetic pleasure that has as its basis simply the survival of the organism? We contend that art conveys more. Goethe's statement "everything is symbol" expresses the essence of art, the power of communication with which it is endowed. Art formulates the intersection between symbol and object that has a comprehensiveness that is universal in application (Heidegger, 2008). The web of relations so evoked is reflective of how art encounters understanding, as relational and unified. Thus the art symbol is generational in its capacity to make evident all to which it relates.

It may be argued that this breadth of relational engagement is seldom achieved. Yet, the implausibility of a simultaneity that may characterize the engagement does not obviate its availability, nor exclude its significance. Extrapolation from symbol to object thus invites the exploration of a world that extensionally lies beyond the form. The revelation of symbol as relational discloses a truth that it conceals in isolation, and the meaning offered for the subject. Its scope, therefore, extends beyond the merely provisional and situates the subject within a landscape of engagement, an engagement that is continually fertile.

As process, engagement confers both orientation and immanency, thereby structuring the definitional characterization to which the subject is processionally conformed. Both constitute the ontological base framing his expression. Situated in a field of engagement the subject is poised, through symbol to object, to forage for an intrinsic meaning whose appeal dictates movement and route, and in which the symbol proffers a presentiment of the end. Along this avenue the aesthetic is determinative of the valued (Scruton, 2009). Engaged, there is the apprehension of symbol in its objective relationality, its exteriority, and its power to situate. United with the external, 
the subject confronts an essence that is reciprocally confirmatory. It is in the provision of this confirmation that is discovered the valuation to which the subject has been directed (Hampson, 2012).

\section{Implications for Quality of Life}

Definitional ambiguities that plague most research intending to evaluate quality of life trace their origin to an empirical approach seeking to assess subjective factors through indirect and non subjective parameters.

As the factors are bereft of subjective meaning for the individual quantification by empirical methodologies is left without conceptual foundation. This situation is not uncommon in studies that purport to assess individual and group dynamics over a broad range of cultural phenomena from business and economic models, to legal studies, to political and social systems, and has led to multiple methodological approaches.

The self reflexive character of the participant in all these studies implicitly underscores valuation of personal meaning for the agent. Interjected into social dynamics, valuation modulates outcome and precipitates policy. To take one example, traditional American jurisprudence underscored by positivist constructs and structured by legal realism claims status of law for social and political judgment, a judgment that is frequently based on a formalized legal reasoning and precedent. Linkage between principle and execution is rarely referenced and doctrinal constructs are reified. The resulting indeterminacy yields a spectrum of interpretations and justifications with option for choice of any or all in subsequent implementation. Absent from deliberations are reference to subjective factors likely to exert causal influence and whose consideration would likely render greater consistency in interpretive range.

Quality of life determinations are particularly sensitive to subjective meaning and its actualization by the individual agent. Without the actualization of a subjectively experienced meaning the agent will derive no "quality" from his "living" experience. Moreover, determinations are subject to combinatorial qualification. Quality of life may embrace a broad conception of summated variables that together yield improved personal status. By the same token it may also situate within one or more parametric considerations. This renders quality of life evaluations subject to more than the sum of its parts, and necessitates a more discrete evaluation than that provided through a utilitarian summation.

Applied extrinsically, definitional interpretations can themselves incorporate a subjective meaning whose origin remains foreign to the agent. Interpretations often bear within themselves a meaning whose significance remains valid primarily for the definer or the assessor. To the extent that this meaning is shared with the agent the definition retains interpretive power. Population domains, subject parameters, and the like delimit applicability. Empirical validation thus claims for the individual an interpretive power that is in reality often nonexistent, elastic in nature, subject to contextual qualification, or modified by definitional preferences.

In view of such considerations the identification of factors through which the agent is endowed with meaning assumes relevance. Such factors may be regarded as causal since the acquisition of personal meaning necessitates an interaction between agent and factor. While the precise form adopted by the factor or the manner of interaction with subject may vary, for quality of life the attribute must personally engage the subject so that he may obtain a meaning that is inaccessible in its absence.

Among the qualities or attributes for which subjective meaning is broadly effectuated is certainly that of artistic beauty. The language of art, expressed through visual, auditory, or conceptual imagery evokes an experiential resonance that is both deeply felt and widely distributed. Throughout history, in nearly all cultures, artistic beauty has been viewed as the sign and substance of civilization. Art has been employed to educate, to heal, and to experience beauty. Indeed, the recognition of art's broad impact prompted the National Endowment for the Arts and US department of Health and Human Services to frame a national research agenda on the relationship between the arts and individual well-being (2011).

Yet, how is this so? The short and direct account is the reciprocal engagement of an apt receiver and a combinative medium. In the first place, the agent possesses an innate potential for reception of the artistic message. Neurally, he engages a cognitively resonant paradigm that is attuned to the art form, one conditioned by the exigencies of personal need. Moreover, beyond the utility of need, he bears within himself desire for personal meaning, and so exercises a resident capacity to instantiate the ontological meaning of the communicated message (Gadamer, 1986).

In the second, the message of art is communicated via the probative medium of quantification. Through the mediation of the quantitative evaluation artistic beauty is made evident.

Quantification effects disclosure, which is constitutive to engagement, and is the medium of encounter. By quantification the image is structured and granted an intelligibility on which is predicated its capacity for transmission. It bridges the gulf of the essentially foreign image to grant it the immediacy of recognition. The task of quantification thus commences with the general hermeneutic of apprehension and conditions the agent for the disclosure of the particular. In its explication of the particular it engages an ongoing evaluative dynamic that is reflective of the compositional construction of each unique art form. Quantification discloses order, a wealth of information, and the unremitting, internal hermeneutic of the constituent dialectical exchange.

It is in its revelation of constituent elements that the peculiarity of the unique image is made comprehensible and granted a contemporaneousness to the agent (Gadamer, 1986), from whom is elicited a reciprocative reply. Nevertheless, the interjection of the particular simultaneously validates the more universal appeal to which the constituent elements relate. Indeed, the more particular is the expression, the deeper and broader the personal resonance that is generally elicited (Pinkaers, 1985).

The constituent elements are not necessarily uniform in their capacity to elicit a reciprocal engagement, however. While order and information wealth may undergird the effectuation of transmission, the sufficiency of the communicative experience necessitates an ongoing mutual evocation between dialectical elements that reside within the image. Art, in fact, is uniquely conditioned by the internal hermeneutic that is mediated by dialectical partners within the art form and which is revealed by quantification.

The role of quantification vis a vis the dialectical operation is manifold.

First, quantification identifies the operationally dialectic 
elements. That is, quantification makes evident the complementary elements that are present in art and which respond one to the other. One may cite, for example, mass and space in architecture, light and dark in the visual arts, or high and low pitch in music. Each element is distinct from, yet also defined by its complement. Indeed each cannot be comprehended in isolation from its dialectical partner. Hence, quantification establishes the oppositional elements that will participate in the dialectic of the art form.

Second, quantification engages the hermeneutic potential of the dialectical exchange. The dialectical constituents support, provoke, and evoke one another (O’Connor, 2003). In a dialectic of material, elementary natural constituents oppose the operation of their partner yet depend on the other for their mutual existence. To take a physical example, atomic structure would cease if the opposing charged particles were neutralized. Likewise, the opposition of complementary sets in the art form sustains the integrity of the form. Architectural form would lose its meaning, for example, if there were no space within which to express form.

The dialectical operation does not merely stabilize the integrity of the art image, however. Dialectical partners respond to each other and are mutually sensitive. Each elicits a mutual response from its partner that enhances its subjective impact and that of the partner. Between the two there is an opposition that is situated by the contrasting elemental composition and which charges their exchange. In effect, the dichotomous relationship establishes an auto-dynamic that is mutually evocative and interminable as the two partners contend one against the other to achieve a balance of tension.

The dialectical operation is brought to internal resolution and superceded by the formation of the unit-pair, the conjoined dialectical partner unit. The pair remains charged and the dynamic operative, but the balanced opposition fundamentally structures the image to situate its focus within the intersection between the two. The shift in focus thus transforms the static elements qualitatively to construct a new dynamic of engagement, and represents the synthesis of the mutually sensitive relations between dialectical partners. This new form qualifies the mutually sensitive elements in their subordination to the dynamic exchange.

The artistic message is thereby communicated by the transformation of the product of the quantitative evaluation into a synthesis of the contending partners. The synthesis constitutes the new level at which the image is situated and represents the teleological focus made accessible through the hermeneutic potential of the raw image. The contending dialectical partners have disclosed the information previously hidden in the preprocessed image and which has lain latent until their evaluative disclosure.

\section{Whither Quality of Life?}

Art's effectiveness for enhancing quality of life is often assessed through quantitative instruments whose focus is the output of the art experience. Yet exploratory commissions decry an absence of confirmatory data and trumpet the need for rigorous research and evidence-based practices intended to document the arts' contributions to human development (National Endowment for the Arts, 2011). The discrepancy between practice and intent is illustrative of a conceptual difficulty at the root of exploration; the empirically evaluated experience is subjective, as it is the quality of life purporting to improve. By illuminating the indirect, quantification eschews the direct. By actuating the experiential engagement of art, however, quantification may more productively explore quality of life (Ratzinger, 2002).

\section{REFERENCES}

Adorno, T. (1970). Asthetische theorie. Ed. Gretel Adorno and Rolf Tiedemann. Frankfurt am Main: Suhrkamp Verlag.

Bergeron, V., \& Lopes, D. (2009). Hearing and seeing musical expression. Philosophy and Phenomenological Research, 78, 1-16. http://dx.doi.org/10.1111/j.1933-1592.2008.00230.x

Birkhoff, G. D. (1933). Aesthetic measure. Cambridge, MA: Harvard University Press.

Bristow, W. (2007). Hegel and the transformation of philo sophical critique. Oxford: Oxford University Press. http://dx.doi.org/10.1093/acprof:oso/9780199290642.001.0001

Chatterjee, A (2010) Neuroaesthetics: A coming of age story. Journal of Cognitive Neuroscience, 23, 53-62.

Chung, M. C., Killingworth, A., \& Nolan, P. (1997). A critique of the concept of quality of life. International Journal of Health Care Quality, 10, 80-84. http://dx.doi.org/10.1108/09526869710166996

Denyer, L. (2004). The principles of design.

Donald, M. (1991). Origins of the Modern Mind: Three stages in the evolution of culture and cognition. Cambridge, MA: Harvard University Press.

Gadamer, H. G. (1986). The relevance of the beautiful. London: Cambridge University Press.

Hampson, P. (2012). "By Knowledge and by Love”: The integrative role of habitus in Christian psychology. Edification, the Transdisciplinary Journal of Christian Psychology, 6, 5-18.

Heidegger, M. (2008). Basic writings: On the origin of the work of art. In D. F. Krell (ed.), 1st Harper Perennial Modern Thought Edition. (pp. 143-212). New York: Harper Collins.

Hildebrand, G. (1999). Origins of architectural pleasure. Berkeley, CA: University of California Press.

Kahneman, D., \& Deaton, A. (2010). High income improves evaluation of life but not emotional well-being. Proceedings of the National Academy of Sciences, 107, 16489-16493. http://dx.doi.org/10.1073/pnas.1011492107

Kim, D (2006). Georg Simmel in translation: Interdisciplinary border crossings in culture and modernity. Cambridge: Cambridge Scholars Press.

LeDoux, J. (2002). The emotional brain revisited. In The synaptic self. (pp. 200-234). New York, NY: Penguin Group.

Leitch, C. M., Hill, F. M., \& Harrison, R. T. (2010). The philosophy and practice of interpretivist research in entrepreneurship. Quality, validation, and trust. Organizational Research Methods, 13, 67-84. http://dx.doi.org/10.1177/1094428109339839

Lorand, R (2000). Ch. 1: What is order? In Aesthetic Order: A Philosophy of Order, Beauty, and Art (pp. 9-16). London: St Edmundsberry Press. http://dx.doi.org/10.4324/9780203468944

Moles, A. (1966). Information theory and esthetic percep tion. Urbana: University of Illinois Press.

Moreno, R., \& Martinez, R. J. (2006) Causality as validity: Some implications for the social sciences. Quality \& Quantity, 42, 597-604. http://dx.doi.org/10.1007/s11135-006-9060-4

National Endowment for the Arts \& US Department of Health and Human Services (2011). The arts and human development: Framing a national research agenda for the arts, lifelong learning, and individual well-being. Washington, DC.

O'Connor, K. (2003). Theories of media: Dialectic. Chicago, IL: University of Chicago Press.

Pinkaers, S. (1985). Les Sources de la Morale Chretienne. Samethode, son contenu, son histoire. Fribourg: Edition Universitaires.

Rappaport, J. (2008). The increasing importance of quality of life. Kansas City, KS: The Federal Reserve Bank of Kansas City Economic Research Department. 


\section{LARRIVEE, A. GINI}

Ratzinger, J. (2002). Congregation for the Doctrine of the Faith The feeling of things, the contemplation of beauty. Message of His Eminence Cardinal Joseph Ratzinger to the Communion and Liberation Meeting at Rimini. Rimini, Italy.

Russell, B. (1919). Ch. 4: The definition of order. In Introduction to Mathematical Philosophy (pp. 46-67). London: George Allen and Unwin, Ltd.

Salingaros, N. (2013). Fractal art and architecture reduce physiological stress. Journal of Biourbanism, 3, 1-3.

Schillinger, J. (1948). The mathematical basis of the arts. New York.

Scruton, R. (2009). Working towards art. British Journal of Aesthetics, 49, 317-325. http://dx.doi.org/10.1093/aesthj/ayp039

Shannon, C. E. (1948). A mathematical theory of communication. Bell System Technical Journal, 27, 379-423.

http://dx.doi.org/10.1002/j.1538-7305.1948.tb01338.x

Solso, R. (2004). The psychology of art and the evolution of the conscious brain. Cambridge, MA: MIT Press.

Spirkin, A. (1983). Dialectical materialism. Moscow: Progress Publishers.

Stanford Encyclopedia of Philosophy (2007). Aristotle. http://plato.stanford.edu/entried/aristotle-categories/

StanfordEncyclopedia of Philosophy (2011). Auguste Comte. http://plato.stanford.edu/entries/comte/

Stanford Encyclopedia of Philosophy (2011). Jurgen Habermas. http://plato.stanford.edu/entries/habermas/

Tersiisky, D. (2004). The elements and principles of design. http://nwrain.net/ tersiisky/design/home.html

Ware, J. E., \& Gandek, B. (1998). Overview of the SF-36 health survey and the international quality of life assessment (IQOLA) project. Journal of Clinical Epidemiology, 51, 903-912.

Zeki, S. (1999). An exploration of art and the brain. Oxford: Oxford University Press. 